\title{
Eulerian spectrum of finite-time Lyapunov exponents in compound channels
}

\author{
Francesco Enrile (1D - Giovanni Besio • Alessandro Stocchino
}

Received: 16 December 2019/ Accepted: 20 July 2020/Published online: 30 July 2020

(C) The Author(s) 2020

\begin{abstract}
Fluid flows reveal a wealth of structures, such as vortices and barriers to transport. Usually, either an Eulerian or a Lagrangian frame of reference is employed in order to detect such features of the flow. However, the two frameworks detect structures that have different properties. Indeed, common Eulerian diagnostics (Hua-Klein and Okubo-Weiss criterion) employed in order to detect vortices do not always agree with Lagrangian diagnostics such as finite-time Lyapunov exponents. Besides, the former are Galilean-invariant whereas the latter is objective. However, both the Lagrangian and the Eulerian approaches to coherent structure detection must show some links under any inertial-frame. Compound channels flows have been accurately studied in the past, both from a Lagrangian and an Eulerian point of view. The features detected do not superimpose: Eulerian vortices do not coincide with barriers to transport. The missing link between the two approaches is here recovered thanks to a spectral analysis.
\end{abstract}

Keywords Lyapunov exponents · River dynamics · Lagrangian Coherent Structures (LCS) · Power

F. Enrile $(\bowtie) \cdot$ G. Besio $~ A$. Stocchino

DICCA, Dipartimento di Ingegneria Civile, Chimica e

Ambientale, Università degli Studi di Genova, Genova,

Italia

e-mail: francesco.enrile@hotmail.it spectral density (PSD) - Eulerian frame of reference · Lagrangian frame of reference

\section{Introduction}

Natural and artificial rivers are quite often characterized by cross-sections composed by a deeper main channel and shallower floodplains. For this reason they are usually referred as "compound channels". Flows of these streams are defined as predominantly horizontal since their horizontal dimensions greatly exceed the vertical one [10].

The analysis of mixing processes in natural streams has already been investigated both from an Eulerian and a Lagrangian point view [19, 21]. Eulerian and Lagrangian approaches are well-known frameworks employed in order to characterize features of the fluid flow as vortices [17] and barriers to transport [4, 6]. In particular, the Eulerian approach uses a fixed frame and focuses on instant velocity fields whereas the Lagrangian approach aims to analyse particle trajectories over fixed time intervals. As a result, both coherent structures and dispersion properties can be analysed under such an approach [7, 13]. Haller [9] pointed out how the two approaches should not be considered interchangeable. However, it is reasonable to assume a link since the basis of these approaches is the same, i.e. the velocity fields. Such a link is still 
lacking in the scientific literature and we argue that it could be fostered through the analysis of the spectral properties of the quantities at hand.

Previous studies aimed to evaluate transport processes in compound channels using Eulerian diagnostic $[3,23,24]$ such as the Okubo-Weiss criterion, usual Lagrangian statistics, e.g. absolute and relative dispersion, and Lagrangian Coherent Structures [5]. These studies were carried out upon surface velocity fields recorded during laboratory experiments via Particle Image Velocimetry (PIV). The present contribution relies upon the same velocity fields since they are validated by the previous cited papers. Stocchino et al. [23] evaluated velocity energy spectra of the streamwise component in compound channels pointing out the presence of an inverse cascade in the energy spectrum depending on the flow conditions. This work aims to clarify whether it is possible to recover the same information by calculating the eulerian spectrum of a classical Lagrangian diagnostic, i.e. the Lyapunov exponents. We would like to find a direct link between Eulerian and Lagrangian frameworks through a spectral analysis.

The paper proceeds with Sect. 2 where the theoretical background is laid. The laboratory experiments are described in Sect. 3. The Results are detailed in Sect. 4. Eventually, the conclusions are drawn in Sect. 5.

\section{Energy spectrum calculations}

Let us consider two initial close particles advected in a two-dimensional turbulence flow. We can introduce Eulerian coordinates as

$$
\boldsymbol{x}=\boldsymbol{\Phi}\left(t ; t_{0}, \boldsymbol{\xi}\right)
$$

where $\boldsymbol{\Phi}$ is the flow map and $\boldsymbol{\xi}$ the Lagrangian coordinates. The trajectories of the particles can be obtained by solving the following set of two ordinary differential equations with appropriate initial conditions

$$
\frac{d \boldsymbol{x}}{d t}=\boldsymbol{u}(\boldsymbol{x}, t)
$$

where $\boldsymbol{u}=(u, v, w)$ is the velocity field. We can evaluate Eq. (2) on a finite time interval $[0, T]$ in order to compute the final distance that particles can experience. Therefore, if we consider as initial conditions $\xi_{0}$ and $\xi_{0}+\epsilon$ we can evaluate the final distance between the two particles applying a linearisation [1]:

$$
\Delta \boldsymbol{x}(T)=\boldsymbol{\Phi}\left(T ; 0, \boldsymbol{\xi}_{0}\right)-\boldsymbol{\Phi}\left(T ; 0, \boldsymbol{\xi}_{0}+\boldsymbol{\epsilon}\right) \approx \nabla \boldsymbol{\Phi}\left(T ; 0, \boldsymbol{\xi}_{0}\right) \boldsymbol{\epsilon}
$$

The magnitude of the final distance can be evaluated as [20]:

$$
\begin{aligned}
|\Delta \boldsymbol{x}(T)| & =\sqrt{\Delta \boldsymbol{x}(T) \cdot \Delta \boldsymbol{x}(T)} \\
& =\sqrt{[\nabla \boldsymbol{\Phi} \Delta \boldsymbol{x}(0)] \cdot[\nabla \boldsymbol{\Phi} \Delta \boldsymbol{x}(0)]}= \\
& =\sqrt{\Delta \boldsymbol{x}(0) \cdot[\boldsymbol{C} \Delta \boldsymbol{x}(0)]}=\sqrt{\boldsymbol{\epsilon} \cdot(\boldsymbol{C} \boldsymbol{\epsilon})}
\end{aligned}
$$

where $\boldsymbol{C}$ is the Cauchy-Green tensor defined as $\boldsymbol{C}=$ $(\nabla \boldsymbol{\Phi})^{T} \nabla \boldsymbol{\Phi}$ where $(\cdot)^{T}$ denotes the transpose. It is possible to prove that matrix $\boldsymbol{C}$ is positive definite and symmetric. Since we analyse $2 \mathrm{D}$ velocity fields, $\boldsymbol{C}$ has two eigenvectors $\boldsymbol{e}_{1}$ and $\boldsymbol{e}_{2}$ associated with two eigenvalues $0<\lambda_{1} \leq \lambda_{2}$, respectively.

Among the infinite directions, in order to experience the maximum separation the two particles must be aligned along the direction pointed out by the eigenvector $\boldsymbol{e}_{2}$ associated with the maximum eigenvalue of the Cauchy-Green tensor. Therefore,

$$
\max |\Delta \boldsymbol{x}(T)| \approx e^{T \sigma_{t_{0}}^{t_{0}+T}}\left|\Delta \widetilde{\boldsymbol{x}}\left(t_{0}\right)\right|
$$

where the superscript $\sim$ indicates alignment with the eigenvector $\boldsymbol{e}_{2}$ and

$$
\sigma_{t_{0}}^{t_{0}+T}(\boldsymbol{x})=\frac{1}{|T|} \log \sqrt{\left(\lambda_{2}\right)}
$$

is the Finite-Time Lyapunov Exponent (FTLE) calculated over the finite-time interval $T$. FTLE can be considered a finite-time average of the maximum expansion rate that a pair of close initial particles advected by the flow can experience in a finite-time interval $T$.

An analysis based on velocity separations $s$ between particles [12] in a two-dimensional turbulent flow with stationary statistics can be enlightening for joining Eulerian and Lagrangian perspectives. The separation velocity $s$ (i.e., the Lagrangian velocity difference) can be written in terms of Eulerian velocity as: 
$\overline{|\boldsymbol{s}(\boldsymbol{\Delta})|^{2}}=\overline{|\boldsymbol{u}(\boldsymbol{x}+\boldsymbol{\Delta}, t)-\boldsymbol{u}(\boldsymbol{x}, t)|^{2}}$

where $\boldsymbol{\Delta}=\left(\Delta_{x}, \Delta_{y}, \Delta_{z}\right)$ is the separation vector with magnitude $|\boldsymbol{\Delta}|=\Delta$ and the overline represents the expected value, $\overline{(\cdot)}=E[(\cdot)]$. If we note that $\Delta(t)=$ $|\Delta \boldsymbol{x}(t)| \approx e^{t \sigma_{0}^{t_{0}+t}}\left|\Delta \boldsymbol{x}\left(t_{0}\right)\right|$ and assuming that the integration is forward in time, we get (the dependence from $t$ is dropped for simplicity):

$\overline{|\boldsymbol{s}(\boldsymbol{\Delta})|^{2}}=\overline{\left|\frac{d \boldsymbol{\Delta}}{d t}\right|^{2}} \approx \overline{\left|\frac{d}{d t} e^{t \sigma_{t_{0}}^{t_{0}+t}}\right|^{2}}\left|\Delta \boldsymbol{x}\left(t_{0}\right)\right|^{2}$

The behavior of separation velocities, i.e. of the structure function, at the varying of the separation has already been analysed by [23]. In the present contribution we would like to analyse the links with Lyapunov exponents. The fundamental relation between the energy spectrum $E(k)$ and the autocorrelation of velocity is written adopting Taylor's hypothesis of frozen turbulence. Therefore, the energy spectrum is obtained in terms of wavenumbers $k$, dividing frequencies by the time and space-averaged surface velocities $U_{s}, k=\frac{f}{U_{s}}$. As a result, we carry out the analysis along the streamwise direction $x[11]$ :

$$
\begin{aligned}
& E(k)=\frac{1}{2 \pi} \int_{-\infty}^{+\infty} R(\Delta) e^{-i k \Delta} d \Delta \\
& R(\Delta)=\int_{-\infty}^{+\infty} E(k) e^{i k \Delta} d k
\end{aligned}
$$

where

$$
R(\Delta)=\overline{u(x+\Delta, t) u(x, t)}
$$

Therefore, we can write (all quantities refer to the streamwise component now):

$$
\overline{|s(\Delta)|^{2}}=\overline{\left|u^{2}(x+\Delta, t)+u^{2}(x, t)-2 u(x+\Delta, t) u(x, t)\right|}
$$

If we recall that:

$$
\overline{u^{2}(x, t)}=\int_{-\infty}^{+\infty} E(k) d k=E_{t}
$$

and we assume independence from a translation of the coordinate system, we also get that:

$$
\overline{u^{2}(x+\Delta, t)}=\int_{-\infty}^{+\infty} E(k) d k=E_{t}
$$

As a result, we could compute the Fourier transform of $\overline{|s(\Delta)|^{2}}$ as:

$$
\begin{aligned}
& F(k)=\frac{1}{2 \pi} \int_{-\infty}^{+\infty} \overline{|s(\Delta)|^{2}} e^{-i k \Delta} d \Delta= \\
&=-\frac{1}{2 \pi} \int_{-\infty}^{+\infty} 2 R(\Delta) e^{-i k \Delta} d \Delta \\
&+\frac{1}{2 \pi} \int_{-\infty}^{+\infty} \overline{u^{2}(x+\Delta, t)} e^{-i k \Delta} d \Delta+ \\
&+\frac{1}{2 \pi} \int_{-\infty}^{+\infty} \overline{u^{2}(x, t)} e^{-i k \Delta} d \Delta= \\
&=-2 E(k)+2 E_{t} \delta(k)=-2 E(k) \text { for } \quad k>0
\end{aligned}
$$

where $\delta(k)$ is the Dirac delta since:

$\int_{-\infty}^{+\infty} e^{-i k x} d x=2 \pi \delta(k)$

As a result, substituting Eq. 8 in 15, we obtain:

$$
\begin{aligned}
F(k) & =\frac{1}{2 \pi} \int_{-\infty}^{+\infty} \overline{|s(\Delta)|^{2}} e^{-i k \Delta} d \Delta \\
& \approx \frac{1}{2 \pi} \int_{-\infty}^{+\infty} \overline{\left|\frac{d}{d t} e^{t \sigma_{t_{0}}^{t_{0}+t}}\right|^{2}} e^{-i k \Delta} d \Delta \propto E(k)
\end{aligned}
$$

We define $F(k)$ as the eulerian spectrum of finite-time Lyapunov exponents since we compute such a spectra from a fixed frame, i.e from an eulerian one. Using Parseval's theorem, it is possible to compute $E(k)$ directly from the streamwise velocity. Thus, we can write:

$$
\begin{aligned}
E(k) & =\frac{1}{2 \pi} \int_{-\infty}^{+\infty} R(\Delta) e^{-i k \Delta} d \Delta \\
& =\lim _{X \rightarrow \infty}\left|\frac{1}{2 \pi} \int_{-X}^{+X} u(x, t) e^{-i k x} d x\right|^{2}=S_{u}
\end{aligned}
$$

where $X$ is the lenght of the domain we take into consideration. Since we cannot extend the above limit to infinity, we have to admit that the above equality holds also for a finite space. The final objective consists in comparing $S_{u}$ and $F(k)$ in order to underline the connection between the Lagrangian and the Eulerian framework as shown in Eq. 17. Energy spectra will be evaluated in Sect. 4 . 


\section{Experimental flow fields}

The present analysis is based on the experimental measurements of the free-surface Eulerian velocity fields described in [5, 23, 24]. Herein, we briefly recall the main characteristics of the apparatus and of the measuring system. The flume was $20 \mathrm{~m}$ long, $60 \mathrm{~cm}$ wide and the trapezoidal cross-section was composed by a central main channel $\left(W_{m c}=20 \mathrm{~cm}\right)$, two lateral flat floodplains $\left(W_{f p}=18 \mathrm{~cm}\right)$ and a transition region $\left(W_{t r}=2.5 \mathrm{~cm}\right)$. Figure 1 shows the cross section of the flume.

Velocity measurements have been performed by means of a two-dimensional Particle Image Velocimetry system on a field of view of $(1.2 \times 0.6) \mathrm{m}^{2}$. The acquisition rate was between 100 and $250 \mathrm{~Hz}$, depending on the flow velocity. Each acquisition was made of a number of frames between 2000 and 4000. Several series of experiments have been carried out spanning a quite large range of values of the main physical parameters. In Table 1 we summarize the experimental conditions, providing the values of the ratio between the main channel water depth $\left(h_{m c}\right)$ and that of the floodplains $\left(h_{f p}\right), r_{h}=h_{m c} / h_{f p}$, and the Froude number, $F r=U_{m} / \sqrt{g R}$, where $R$ is the hydraulic radius, $g$ is gravity and $U_{m}$ is the peak velocity in the main channel. Moreover, $S$ represents the longitudinal bed slope. The experiments under analysis are the same as those studied in [5] in order to allow for a direct comparison with Lagrangian Coherent Structures.

We keep the same distribution of the flow regimes depending on $r_{h}$ introduced in [15] and used in [23]. As pointed out by [15] three different flow regimes can be identified depending on the value of $r_{h}$. For $r_{h}>3$ the
Table 1 Main parameters of the experiments under analysis

\begin{tabular}{cllll}
\hline Exp. & $r_{h}(-)$ & $\operatorname{Fr}(-)$ & $S(-)$ & $\operatorname{Re} \times 10^{4}(-)$ \\
\hline 201 & 4.16 & 0.60 & 0.0032 & 20.1 \\
105 & 2.15 & 1.05 & 0.0064 & 84.9 \\
205 & 2.57 & 0.69 & 0.0032 & 41.4 \\
207 & 2.26 & 0.73 & 0.0032 & 51.8 \\
213 & 1.68 & 0.82 & 0.0032 & 130.1 \\
\hline
\end{tabular}

flow is defined as "Shallow". In this case, intense velocity gradients occur at the transition between the main channel and the floodplains, leading to a strong shearing and a generation of vorticity associated with the flow depth jump [22]. For values of $r_{h}<2$, the flow is defined as "Deep", characterized by a weaker shear in the transition region. The flow depth jump, in this case, is unable to greatly influence the free-surface flow. Finally, "Intermediate flows" are defined when $2<r_{h}<3$.

\section{Results and discussion}

Compound channel flows present different behaviours depending on Froude number (supercritical or subcritical conditions) and on the depth jump (shallow, intermediate and deep flows). In particular, an inverse energy cascade can be observed for high Froude number flows [16] and in shallow and intermediate flows [23].

Evidence of the presence of an inverse energy cascade occurring in shallow flow conditions can be found by means of the dimensionless Eulerian power

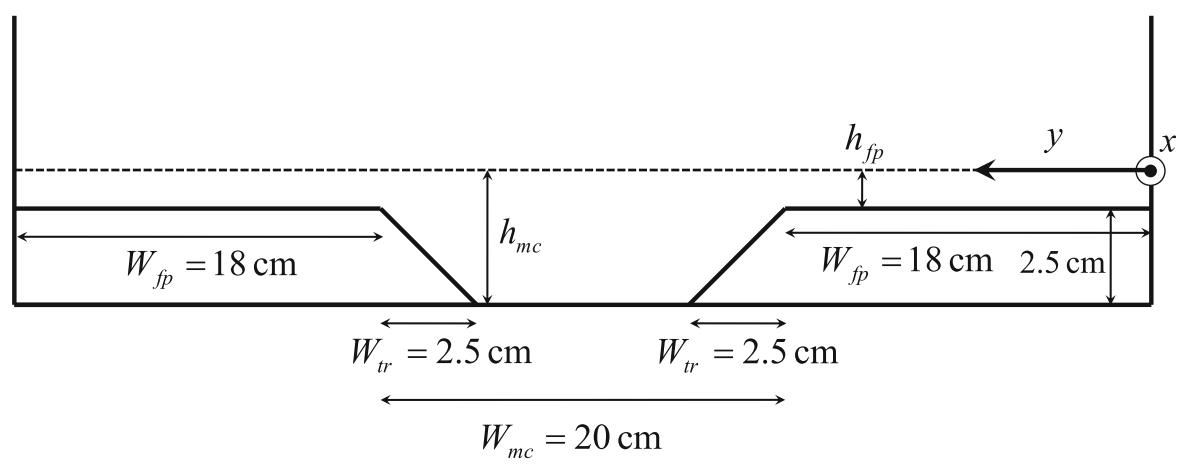

Fig. 1 Sketch of the cross section of the flume 
spectral density (PSD) function $S_{u}(k L)$ of the streamwise velocity component, where the wavenumber $k$ has been made dimensionless using $W_{t r}=L$ [23]. The PSD functions have been normalized with the friction velocity $u_{*}$ defined as $u_{*}=\sqrt{g h S}$. Owing to the limited size of the velocity series in space, the computation of the PSD has been carried out in terms of frequency $S_{u}(f)$ and, subsequently, evaluated in terms of wavenumbers by dividing each frequency by the time and space-averaged surface velocity $U_{s}$, $k=f / U_{s}$, under the assumption of "frozen turbulence", as suggested by [16].

The presence of an inverse energy cascade can be further assessed by analysing the Kolmogorov $S_{3}$ structure function [25]. Such a function can be defined as $S_{3}=\left\langle\delta u(\Delta)^{3}\right\rangle$, with $\delta u(\Delta)=u(x+\Delta)-u(x)$. The evaluation of the Kolmogorov structure function is carried out in agreement with [16]. The transverse component of velocity has at least one order of magnitude less than the streamwise component since the flow is mainly unidirectional. Thus, structure functions and spectra reveals the behaviour of the streamwise component.

For all experiments, the spatial structure functions were negative at small separations 4 . Such a behaviour is expected, in general, for reasonably small scales where energy transfer is directed from larger eddies to smaller eddies, in agreement with the classical picture of open channel flow. With an increase in $\Delta$ the structure functions change their sign

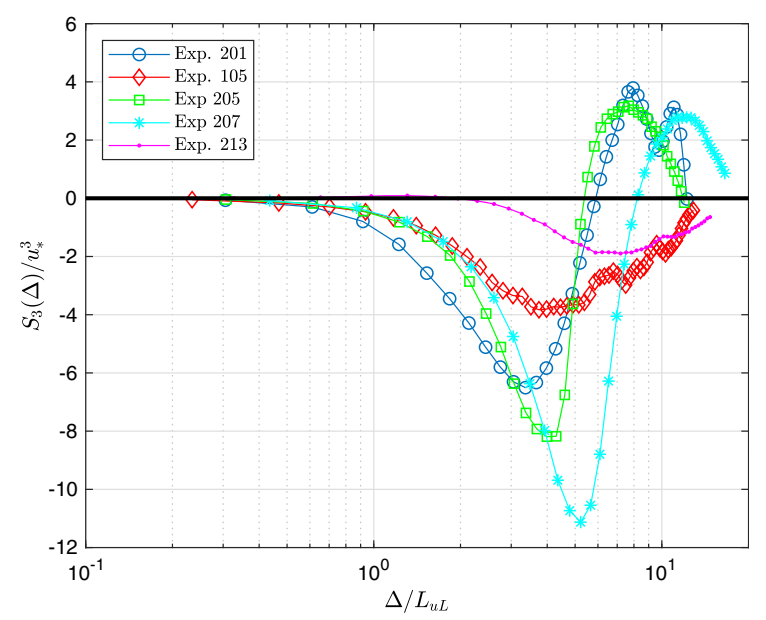

Fig. $2 S_{3}$ Kolmogorov structure function normalized with the friction velocity. Lengths $\Delta$ are made non-dimensional through the longitudinal Lagrangian Integral Length Scale $L_{u L}$ from negative to positive suggesting an inverse energy cascade at larger scales. Figure 2 shows the structure function for the analysed experiments. $S_{3}$ is made nondimensional with the friction velocity whereas separations $\Delta$ with the Lagrangian Integral Length Scale $L_{u L}$ [8]. As expected, the shallow case (exp. 201) and the intermediate subcritical cases (exp. 205 and 207) show an inverse energy cascade. The deep case and the intermediate supercritical cases (exp. 213 and 105, respectively) do not show such an inversion. Details about the root causes of such an inversion are detailed in [23]. Summarising such results, it is possible to argue that in shallow flows, i.e. when the depth jump is great, eulerian macro-vortices are triggered by the mechanism described by [22] and the injection scale is the depth jump itself. These flows present an inverse energy cascade. For Intermediate flows, it seems that only for subcritical conditions does an inverse energy cascade survive (the injection scale always related to the flow depth jump as in the shallow flow conditions), whereas only an enstrophy cascading process occurs for supercritical flows. In deep flows the influence of the depth jump is low and only a typical direct energy cascade is present. Therefore, considerations about the structure functions are here reported in order to strengthen our conclusions circa the spectra of the Lyapunov exponents.

The evaluation of eulerian spectra of finite-time Lyapunov exponents $F$ is compared with the PSD of the streamwise component of the velocity $S_{u}$ in the following pictures. The spectra are calculated as the absolute value of the Fourier Transforms and therefore they have the same monotonicity, instead of the opposed one of Eq. 15. It is possible to appreciate the same scalings, showing the desired link between the Eulerian and the Lagrangian approaches. The analysis of the spectral properties is a unique way to underline that the energy content is preserved adopting special diagnostic to capture the pattern of the flow, for example through LCS. This result guarantees that Eulerian and Lagrangian frames do show a link and that the use of Lyapunov exponents do not alter the properties of the flow keeping unaltered the turbulence properties. The computations are carried out over a domain $X=1.2 \mathrm{~m}$ long and considering surface velocities recorded along the depth jump. The final spectra reported represent a space-averaged spectra that are commonly adopted in geophysical studies also from large-scale numerical modelling [14]. Figure 3 


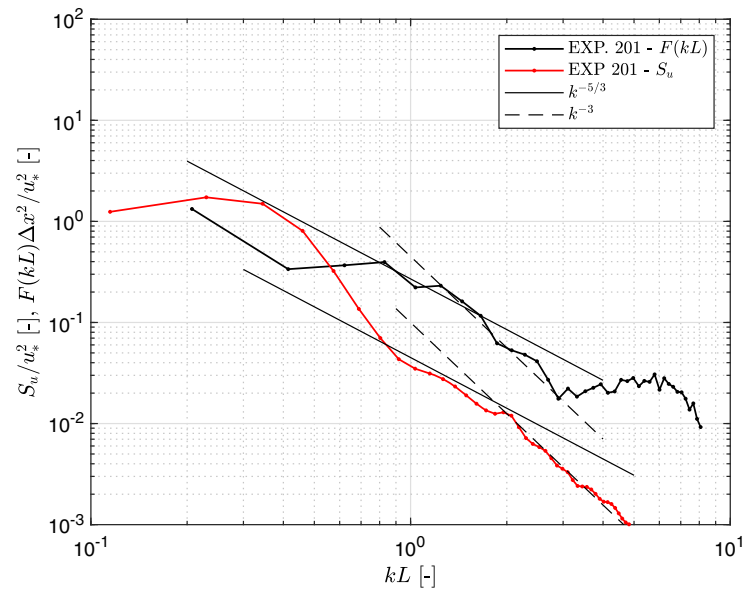

Fig. 3 Experiment 201, subcritical shallow flow. An inverse energy cascade is shown with the typical powers of $-5 / 3$ and -3 . Such a trend is also idenfied in Fig. 2

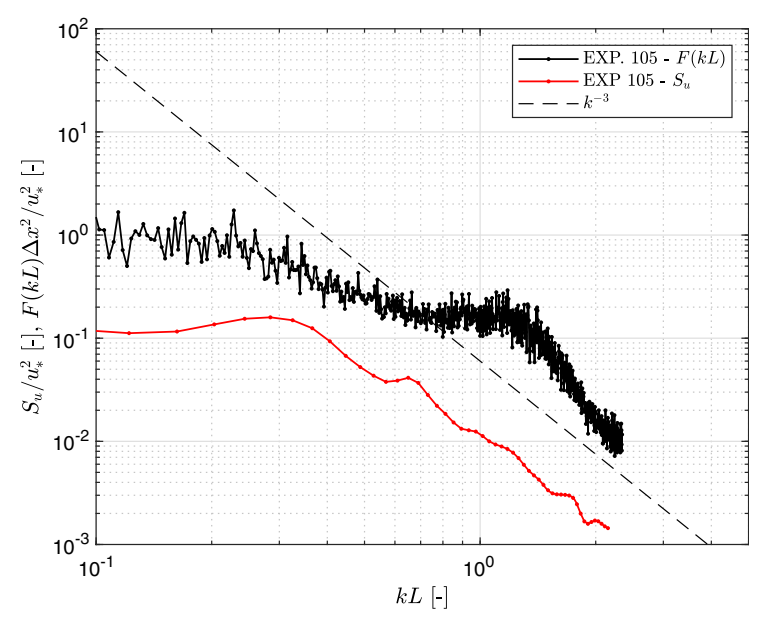

Fig. 4 Experiment 105, supercritical intermediate flow. A direct energy cascade is present

shows an inverse energy cascade as predicted by the structure function. Both $F$ and $S_{u}$ shows the same trends. Figure 4 shows a direct enstrophy cascade with a trend proportional to $k^{-3}$. Analogous result can be recovered for Fig. 5. Conversely, Figs. 6 and 7 show an inverse energy cascade analogous to Fig. 3. In general, the PSD $S_{u}$ of the streamwise component of the velocity shows a quite smooth trend since we employed a Welch's windowing. On the contrary, the eulerian spectrum of finite-time Lyapunov exponents $F$ shows noisier signals since we carried out the computation of the Fourier Transform shown in Eq. 17 directly applying a Fast Fourier Transform algorithm.

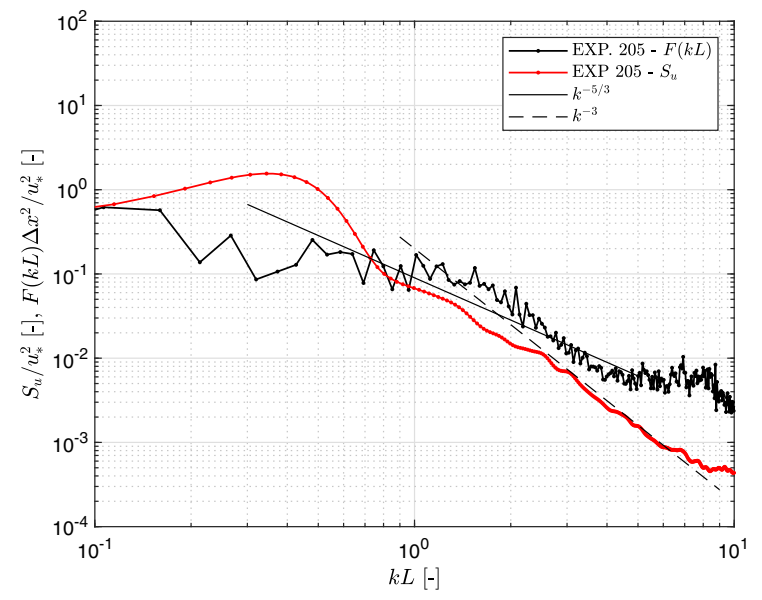

Fig. 5 Experiment 205, subcritical intermediate flow. Analogously to Fig. 3 a inverse energy cascade is present

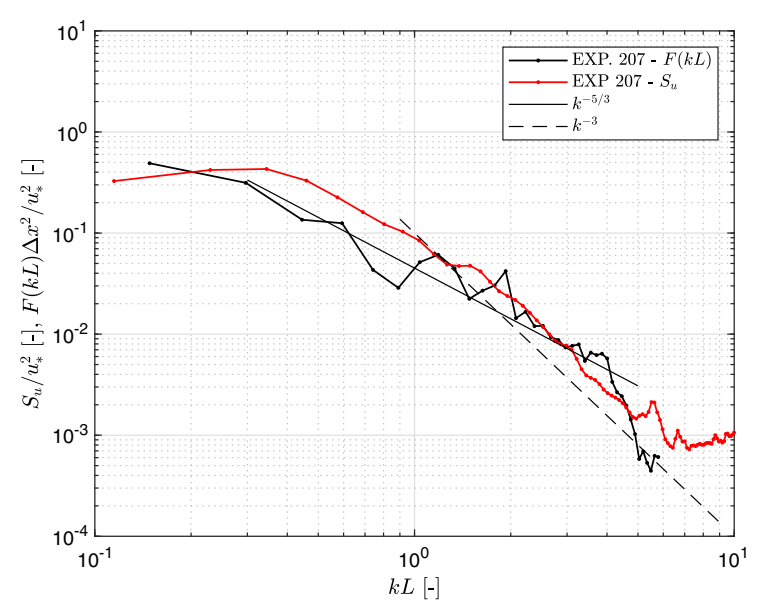

Fig. 6 Experiment 207, subcritical intermediate flow. Similar to Fig. 6

It is worth to remember that this analysis is carried out on experimental velocity fields whereas typical results in scientific literature are deduced from numerical simulations $[2,26]$.

\section{Conclusions}

Compound channels have been studied in several research works pointing out the spectral properties of such flows. Among the vast literature, it is worth to cite $[16,18,23]$ where under some circumstances the structure of the surface resembles that of two-dimensional turbulence with an inverse energy cascade. This 


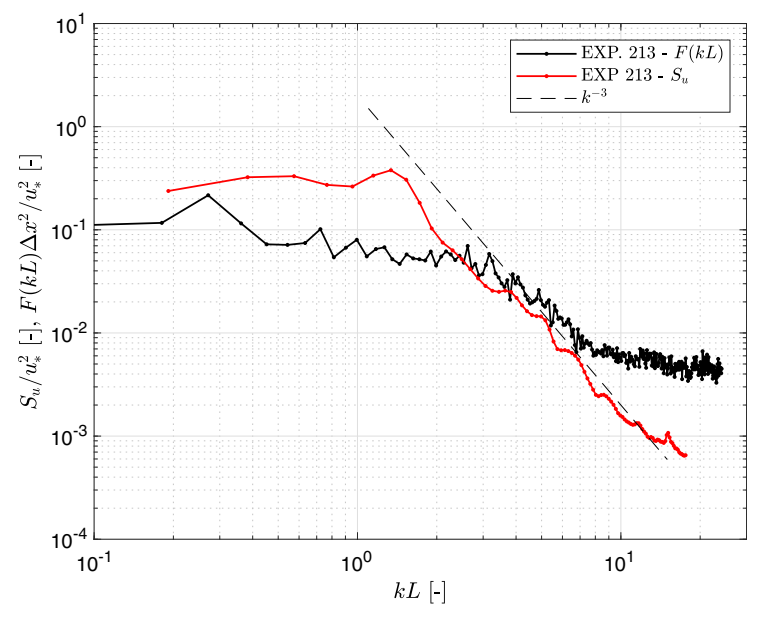

Fig. 7 Experiment 213, supercritical deep flow. This case is similar to Fig. 4

behaviour occurs when the flow is in subcritical conditions $(F r<1)$ and enough shallow $\left(r_{h}<3\right)$. A recent contribution [5] aimed at evaluating Lagrangian Coherent Structures on such flows and concluded stating that "a link between the two frameworks (Eulerian and Lagrangian) based on the spectral properties of Eulerian velocity fields and FTLE fields would then be desirable". The present work fills this need and proves that it is possible to find a connection thanks to an analysis based on energy considerations.

Adopting a Lagrangian framework can have several advantages. Primarily, the ability of locating barriers to transport which are difficult to locate in an Eulerian frame. However, a connection with the Eulerian frame seems to be missing. This work aims at linking the Eulerian and the Lagrangian frame of reference comparing the spectral properties of the streamwise component of the velocity with those of a classic Lagrangian quantity, i.e. the finite-time Lyapunov exponent. This link is found and is presented through energy spectra whose behaviour is in agreement with the Kolmogorov structure function $S_{3}$. Such results also validate the use of Eulerian and Lagrangian diagnostic since they preserve the turbulence properties of the flow. These results are Galilean-invariant. For any inertial-frame of reference such results are valid, apart for the contribution of the mean velocity. Indeed, the characteristics of the flow are preserved in both Eulerian and Lagrangian frameworks, reinforcing the known results and showing how they not only unveil possible features of the flow (i.e. barriers to transport and vortical structures), but carry also the fingerprints of turbulence with them.

Acknowledgements Open access funding provided by Università degli Studi di Genova within the CRUI-CARE Agreement. We would like to thank Dr. Daniele Lagomarsino Oneto and Mr. Stefano Pastorino for the useful discussions.

\section{Compliance with ethical standards}

Conflict of interest The authors declare that they have no conflict of interest.

Open Access This article is licensed under a Creative Commons Attribution 4.0 International License, which permits use, sharing, adaptation, distribution and reproduction in any medium or format, as long as you give appropriate credit to the original author(s) and the source, provide a link to the Creative Commons licence, and indicate if changes were made. The images or other third party material in this article are included in the article's Creative Commons licence, unless indicated otherwise in a credit line to the material. If material is not included in the article's Creative Commons licence and your intended use is not permitted by statutory regulation or exceeds the permitted use, you will need to obtain permission directly from the copyright holder. To view a copy of this licence, visit http://creativecommons.org/licenses/by/4.0/.

\section{References}

1. Allshouse MR, Peacock T (2015) Refining finite-time lyapunov exponent ridges and the challenges of classifying them. Chaos 25(8):087410

2. Alvenius K, Johansson AV (2000) LES computations and comparison with kolmogorov theory for two-point pressurevelocity correlations and structure functions for globally anisotropic turbulence. J Fluid Mech 403:23-36

3. Besio G, Stocchino A, Angiolani S, Brocchini M (2012) Transversal and longitudinal mixing in compound channels. Water Resour Res 48(12)

4. Boffetta G, Lacorata G, Redaelli G, Vulpiani A (2001) Detecting barriers to transport: a review of different techniques. Phys D Nonlinear Phenom 159:58-70

5. Enrile F, Besio G, Stocchino A (2018a) Shear and shearless lagrangian structures in compound channels. Adv Water Resour 113:141-154

6. Enrile F, Besio G, Stocchino A, Magaldi M, Mantovani C, Cosoli S, Gerin R, Poulain P (2018b) Evaluation of surface lagrangian transport barriers in the gulf of trieste. Cont Shelf Res 167:125-138

7. Enrile F, Besio G, Stocchino A, Magaldi MG (2019) Influence of initial conditions on absolute and relative dispersion in semi-enclosed basins. PLoS One 14(7):1-12

8. Fischer HB, List JE, Koh CR, Imberger J, Brooks NH (1979) Mixing in inland and coastal waters. Academic Press, New York

9. Haller G (2015) Lagrangian coherent structures. Ann Rev Fluid Mech 47:137-162 
10. Jirka G (2001) Large scale flow structures and mixing processes in shallow flows. J Hydraul Res 39:567-573

11. Kundu PK, Cohen IM, Dowling DR (eds) (2012) Chapter 13-geophysical fluid dynamics, 5th edn. Academic Press, Boston

12. LaCasce J (2008) Statistics from lagrangian observations. Progr Oceanogr 77:129

13. LaCasce J, Bower A (2000) Relative dispersion in the subsurface north atlantic. J Mar Res 58:863-894

14. Magaldi MG, Haine TW (2015) Hydrostatic and non-hydrostatic simulations of dense waters cascading off a shelf: the east greenland case. Deep Sea Res Part I Oceanogr Res Pap 96:89-104

15. Nezu I, Onitsuka K, Iketani K (1999) Coherent horizontal vortices in compound open channel flows. In: Singh VP, Seo IW, Sonu JH (eds) Hydraulic modeling. Water Resources Pub, Colorado, USA, pp 17-32

16. Nikora V, Nokes R, Veale W, Davidson M, Jirka G (2007) Large-scale turbulent structure of uniform shallow freesurface flows. Environ Fluid Mech 7:159-172

17. Okubo A (1970) Horizontal dispersion of floatable particles in the vicinity of velocity singularities such as convergences. Deep Sea Res 17:445-454

18. Proust S, Fernandes JN, Leal JB, Rivire N, Peltier Y (2017) Mixing layer and coherent structures in compound channel flows: effects of transverse flow, velocity ratio, and vertical confinement. Water Resour Res 53(4):3387-3406
19. Rowiński P, Radecki-Pawlik A (2015) Rivers-physical, fluvial and environmental processes. Springer, Berlin

20. Shadden SC, Lekien F, Marsden JE (2005) Definition and properties of Lagrangian coherent structures from finitetime Lyapunov exponents in two-dimensional aperiodic flows. Phys D Nonlinear Phenom 212:271-304

21. Socolofksy S, Jirka G (2004) Large-scale flow structures and stability in shallow flows. J Environ Eng Sci 3:451-462

22. Soldini L, Piattella A, Brocchini M, Mancinelli A, Bernetti $R$ (2004) Macrovortices-induced horizontal mixing in compound channels. Ocean Dyn 54:333-339

23. Stocchino A, Besio G, Angiolani S, Brocchini M (2011) Lagrangian mixing in straight compound channel. J Fluid Mech 675:168-198

24. Stocchino A, Brocchini M (2010) Horizontal mixing of quasi-uniform straight compound channel flows. J Fluid Mech 643:425-435

25. Van De Water W, Herweijer JA (1999) High-order structure functions of turbulence. J Fluid Mech 387:3-37

26. Xie J-H, Bühler O (2018) Exact third-order structure functions for two-dimensional turbulence. $\mathrm{J}$ Fluid Mech 851:672-686

Publisher's Note Springer Nature remains neutral with regard to jurisdictional claims in published maps and institutional affiliations. 